\title{
ON THE FURTHER REFINEMENT OF SOME OPERATOR INEQUALITIES FOR POSITIVE LINEAR MAP
}

\author{
Changsen YANG AND YU Li*
}

Abstract. In this paper, some further improvements of operator inequalities are given at the base of Yang et al.'s recent work [Filomat 32:12 (2018), 4333-4340] and [Math. Slovaca 69 (2019), 919-930] for positive linear map. Besides, the corresponding multiple-term refinements for scalars and operators are shown as well.

Mathematics subject classification (2020): Primary 47A63; Secondary 47A30.

Keywords and phrases: Positive linear map, operator inequality, multiple-term refinements.

\section{REFERENCES}

[1] R. Bhatia, Matrix Analysis, GTM 169, Springer-Verlag, New York, 1997.

[2] R. Bhatia, F. Kittaneh, Notes on matrix arithmetic-geometric mean inequalities, Linear Algebra Appl., 308 (2000), 203-211.

[3] R. Bhatia, Positive Definite Matrices, Princeton University Press, Princeton, 2007.

[4] T. Furuta, J. Mićić Hot, J. Pečarić, Mond-Pečarić Method in Operator Inequalities, Element, Zagreb, 2005.

[5] R. A. Horn, C. R. Johnson, Topics in Matrix Anlysis, Cambridge University Press, 1991.

[6] F. Kubo, T. Ando, Means of positive operators, Math. Ann., 264 (1980), 205-224.

[7] S. KIM, H. LEE, Relative operator entropy related with the spectral geometric mean, Anal. Math. Phys., 5 (2015), 233-240.

[8] P. KóRus, A refinement of Young's inequality, Acta Math. Hungar., 153 (2017), 430-435.

[9] M. LIN, Squaring a reverse AG-GM inequality, Studia Math., 215 (2013), 187-194.

[10] M. Lin, On an operator Kantorovich inequality for positive linear map, J. Math. Anal. Appl., 402 (2013), 127-132.

[11] Y. REN, P. LI, Further refinements of reversed AM-GM operator inequalities, J. Inequal. Appl., 2020, $1(2020)$.

[12] M. Sababhen, D. Choi, A completed refinement of Young's inequality, J. Math. Anal. Appl., 440 (2016), 379-393.

[13] C. YAng, Y. GAO, F. LU, Some refinements of Young type inequality for positive linear map, Math. Slovaca, 69, 4 (2019), 919-930.

[14] C. YANG, F. LU, Improving some operator inequalities for positive linear maps, Filomat, 32, 12 (2018), 4333-4340.

[15] X. ZHAO, L. LI, H. ZUO, Further improved Young inequalities for operator and matrices, J. Math. Inequal., 11 (2017), 1023-1029. 